\title{
Cannabidiol (CBD) content in vaporized cannabis does not prevent tetrahydrocannabinol (THC)-induced impairment of driving and cognition
}

\author{
Thomas R. Arkell ${ }^{1,2,3} \cdot$ Nicholas Lintzeris $^{3,4} \cdot$ Richard C. Kevin $^{1,2,5} \cdot$ Johannes G. Ramaekers $^{6} \cdot$ Ryan Vandrey $^{7}$. \\ ${\text { Christopher } \text { Irwin }^{8} \cdot \text { Paul S. Haber }}^{3,9} \cdot$ Iain S. McGregor ${ }^{1,2,5}$
}

Received: 17 January 2019 / Accepted: 9 April 2019 / Published online: 1 May 2019

(C) The Author(s) 2019

\begin{abstract}
Background The main psychoactive component of cannabis, delta-9-tetrahydrocannabinol (THC), can impair driving performance. Cannabidiol (CBD), a non-intoxicating cannabis component, is thought to mitigate certain adverse effects of THC. It is possible then that cannabis containing equivalent $\mathrm{CBD}$ and THC will differentially affect driving and cognition relative to THC-dominant cannabis. Aims The present study investigated and compared the effects of THC-dominant and THC/CBD equivalent cannabis on simulated driving and cognitive performance.

Methods In a randomized, double-blind, within-subjects crossover design, healthy volunteers $(n=14)$ with a history of light cannabis use attended three outpatient experimental test sessions in which simulated driving and cognitive performance were assessed at two timepoints (20-60 min and 200-240 min) following vaporization of $125 \mathrm{mg}$ THC-dominant (11\% THC; < 1\% CBD), THC/CBD equivalent (11\% THC, $11 \%$ CBD), or placebo (<1\% THC/CBD) cannabis.

Results/outcomes Both active cannabis types increased lane weaving during a car-following task but had little effect on other driving performance measures. Active cannabis types impaired performance on the Digit Symbol Substitution Task (DSST), Divided Attention Task (DAT) and Paced Auditory Serial Addition Task (PASAT) with impairment on the latter two tasks worse with THC/CBD equivalent cannabis. Subjective drug effects (e.g., "stoned") and confidence in driving ability did not vary with CBD content. Peak plasma THC concentrations were higher following THC/CBD equivalent cannabis relative to THC-dominant cannabis, suggesting a possible pharmacokinetic interaction.

Conclusions/interpretation Cannabis containing equivalent concentrations of CBD and THC appears no less impairing than THC-dominant cannabis, and in some circumstances, CBD may actually exacerbate THC-induced impairment.
\end{abstract}

Keywords Cannabis $\cdot$ Tetrahydrocannabinol $\cdot$ Cannabidiol $\cdot$ THC $\cdot$ CBD $\cdot$ Driving $\cdot$ Cognition

Electronic supplementary material The online version of this article (https://doi.org/10.1007/s00213-019-05246-8) contains supplementary material, which is available to authorized users.

Iain S. McGregor

iain.mcgregor@sydney.edu.au

1 Lambert Initiative for Cannabinoid Therapeutics, The University of Sydney, Sydney, New South Wales, Australia

2 Brain and Mind Centre, The University of Sydney, Sydney, New South Wales, Australia

3 Faculty of Medicine, Central Clinical School, The University of Sydney, Sydney, New South Wales, Australia

4 The Langton Centre, Drug and Alcohol Services, South East Sydney Local Health District, NSW Health, Sydney, New South Wales, Australia
5 Faculty of Science, School of Psychology, The University of Sydney, Sydney, New South Wales, Australia

6 Faculty of Psychology and Neuroscience, Maastricht University, Maastricht, The Netherlands

7 Johns Hopkins University School of Medicine, Baltimore, MD, USA

8 School of Allied Health Sciences, Griffith University, Gold Coast, Australia

9 Drug Health Services, Royal Prince Alfred Hospital, Camperdown, New South Wales, Australia 


\section{Introduction}

With the growing worldwide trend towards the decriminalization of recreational and medicinal cannabis use, there has been a renewed focus on the risks associated with driving under the influence of cannabis (Ramaekers 2018; Capler et al. 2017). Epidemiological studies suggest that cannabis intoxication produces a modest increase in the risk of being involved in a crash (Rogeberg and Elvik 2017; Rogeberg and Elvik 2016) although not the risk of being seriously injured or killed (Schulze et al. 2012). On-the-road and laboratory studies of driving performance consistently show that cannabis tends to impair driving-related skills and cognitive functions in a dosedependent manner (Veldstra et al. 2015; Bosker et al. 2012; Lamers and Ramaekers 2001; Downey et al. 2013; Hartman et al. 2015).

These experimental studies of driving performance have typically involved administration of smoked cannabis containing $\Delta^{9}$-tetrahydrocannabinol (THC), or pharmaceutical THC given in a capsule form (e.g., dronabinol) (Veldstra et al. 2015; Bosker et al. 2012; Lamers and Ramaekers 2001; Downey et al. 2013; Hartman et al. 2015; Papafotiou et al. 2005). However, as the therapeutic effects of the nonintoxicating cannabinoid cannabidiol (CBD) become more apparent (Zhornitsky and Potvin 2012; Cuba et al. 2017; Mannucci et al. 2017), it is likely that community use of medicinal cannabis products containing both THC and CBD will become increasingly common.

There is speculation, and some evidence, that CBD may minimize some of the negative side effects associated with THC and enhance therapeutic efficacy (Hindocha et al. 2015; Martin-Santos et al. 2012; Bhattacharyya et al. 2010; Russo and Guy 2006). CBD has sometimes been found to lessen the "euphoria" associated with cannabis intoxication (Dalton et al. 1976), and attenuate THC-induced attentional bias towards food and drug-related stimuli (Morgan et al. 2010b). CBD can lessen THC-induced impairment of facial emotion recognition (Hindocha et al. 2015) and improve such recognition when administered alone. Naturalistic studies suggest that cannabis users consuming higher CBD products experience fewer psychotic experiences (Schubart et al. 2011), fewer positive schizophrenia-like symptoms (Morgan and Curran 2008), and less memory-impairment (Morgan et al. 2010a).

Other studies, however, have failed to observe modulatory effects of CBD on THC subjective drug effects (e.g., being "stoned") when the CBD is smoked (Morgan et al. 2010a; Ilan et al. 2005), vaporized (Hindocha et al. 2015), or administered orally (Roser et al. 2008; Juckel et al. 2007; Hollister and Gillespie 1975; Haney et al. 2016). In one study, pretreatment with CBD (up to $800 \mathrm{mg}$ oral) did not alter the physiological or subjective effects of smoked THCdominant cannabis (Haney et al. 2016). Preclinical studies indicate that $\mathrm{CBD}$ can even sometimes potentiate some of the behavioral and cognitive effects of THC, possibly by way of a pharmacokinetic interaction whereby CBD potentiates blood THC levels (Boggs et al. 2018; Klein et al. 2011).

The above inconsistencies highlight the need for human studies that systematically compare the effects of different cannabis chemovars ("strains") containing varying amounts of THC and CBD. In jurisdictions where medicinal and/or recreational cannabis is widely available, users often have a choice of hundreds of such types of cannabis, which vary with respect to THC and CBD content. This study therefore sought to compare the subjective, cognitive, and driving-related effects of vaporized THC-dominant $(11 \%$ THC, $<1 \%$ CBD [hereafter "THC"]), THC/CBD-equivalent (11\% THC, 11\% CBD [hereafter "THC/CBD"]) and placebo $(<1 \%$ THC; $<1 \%$ CBD) cannabis.

\section{Methods}

\section{Study design and procedures}

This double-blind, within-subjects crossover study included three experimental sessions that were scheduled at least 7 days apart to avoid carryover effects. Participants received the three treatments (one per session) in a randomized and counterbalanced order. The randomization schedule was created by an independent researcher, and only the study pharmacist had access to it. Between 1 and 4 weeks prior to the first session, participants attended an orientation session in which they practiced the driving simulation and cognitive tasks. Practice was continued until participants demonstrated competence in each task. Participants were instructed to abstain from illicit drugs for the duration of the study (i.e., from the time of study enrollment until the final session) and from alcohol on the night before research sessions, and to maintain any use of regular medications. Participants were also instructed to consume no more than their regular caffeine intake on the morning of research sessions.

\section{Participants}

Participants were healthy adults with a history of infrequent cannabis use. Inclusion criteria were aged 18-65 years, selfreported cannabis consumption $\leq 2$ times/week in the previous 3 months and $\geq 10$ lifetime exposures, and possession of and minimum 1-year driving on an unrestricted Australian license (i.e., $>4$ years total driving experience). Exclusion criteria included current mood disorder, lifetime major psychiatric illness, history of clinically significant adverse response to previous cannabis exposure, any moderate or severe substance use disorder as assessed by an addiction medicine specialist, pregnant/nursing, interest in treatment to reduce cannabis use, current use of medications known to affect driving, active 
hypertension, cardiovascular disease, or chronic pulmonary disease. Volunteers were recruited through online advertisement, social media (e.g., Facebook), and word of mouth. After an initial phone screen, participants meeting inclusion/ exclusion criteria were invited to attend a medical screen which involved a detailed medical and psychiatric evaluation. All participants gave written informed consent prior to study enrollment. All procedures were approved by the Sydney Local Health District (RPAH Zone) Human Research Ethics Committee and were in accordance with the Declaration of Helsinki. The trial was listed on the Australia New Zealand Clinical Trials Registry (No. 12616000414415).

\section{Experimental sessions}

The order of events during research sessions is presented in Fig. 1. Participants arrived at the clinical research unit at 09:00 on the morning of research sessions. Nil breath alcohol concentration $(\mathrm{BrAC})$ was confirmed via breathalyzer (Alcotest 5510, Draeger, Lübeck, Germany), and oral fluid was screened (DrugWipe 5s, Securetec, Neubiberg, Germany) to rule out recent drug use. Participants testing positive for any drug (cannabis, cocaine, opiates, or amphetamines/MDMA/ methamphetamines) were sent home and the session was rescheduled. Participants completed a baseline questionnaire at the start of each session which asked about recent use of drugs, alcohol and caffeine, adverse events since the previous session, and perceived sleep quality during the previous night. Baseline cognitive task performance and subjective drug effects were assessed $30 \mathrm{~min}$ prior to dosing.

Participants inhaled $125 \mathrm{mg}$ THC (11\% THC, < 1\% CBD), THC/CBD (11\% THC, $11 \% \mathrm{CBD})$, or placebo $(<1 \% \mathrm{THC},<$ $1 \% \mathrm{CBD}$ ) cannabis (Tilray, BC, Canada) via vaporization at $200{ }^{\circ} \mathrm{C}$ (Mighty Medic, Storz \& Bickel, Tuttlingen, Germany) over $5 \mathrm{~min}$ according to a standardized procedure (inhale $3 \mathrm{~s}$, hold $3 \mathrm{~s}$, exhale, and rest $30 \mathrm{~s}$ ). If vapor was still visible in exhaled breath at $5 \mathrm{~min}$, then this procedure was continued until vapor was no longer visible.

\section{Blood collection and plasma cannabinoid levels}

Blood was collected via indwelling peripheral venous catheter into purple-top (EDTA) Vacutainer® tubes (Becton, Dickinson and Company, Franklin Lakes, NJ) 20 min prior to and 10-, 60-, 120-, and 180-min post-inhalation. The blood was centrifuged at $1228 \times \mathrm{g}$ for $10 \mathrm{~min}$ and the supernatant plasma was decanted and stored in $3.6-\mathrm{mL}$ Nunc ${ }^{\circledR}$ cryotubes (Thomas Scientific, Swedesboro, NJ) at $-80{ }^{\circ} \mathrm{C}$ until analysis. Plasma was subsequently thawed for analysis via liquid chromatography-tandem mass spectrometry (LC-MS/MS) according to previously published methods (Kevin et al. 2017; Schwope et al. 2011). Duplicate $200-\mu \mathrm{L}$ plasma aliquots were fortified with an internal standard mixture containing $d_{3}$-THC, $d_{3}$-THC$\mathrm{COOH}$, and $d_{3}$-11-OH-THC. Duplicate calibrator samples were prepared with cannabinoid-free plasma (obtained from the Red Cross), spiked with appropriate amounts of a standard mixture of THC, 11-OH-THC, THC-COOH, CBD, and internal standards to generate a standard curve and quality control samples for each analyte. All samples were diluted $1: 1$ in $0.1 \%$ formic acid in water, and analytes were extracted using $400 \mu \mathrm{L}$ capacity ISOLUTE SLE+ supported liquid extraction columns (Biotage, Sydney, Australia). The analytes were eluted with $500 \mu \mathrm{L}$ dichloromethane, $300 \mu \mathrm{L}$ ethyl acetate, and $1.2 \mathrm{~mL}$ methyl tert-butyl ether. The eluate was evaporated under a gentle stream of nitrogen without heating, and analytes were reconstituted in $100 \mu \mathrm{L}$ of $40: 60$ acetonitrile and $0.1 \%$ formic acid in water, transferred to 2-mL autosampler vials fitted with $100-\mu \mathrm{L}$ glass inserts, and placed in the LC-MS/MS autosampler held at $4{ }^{\circ} \mathrm{C}$. Chromatographic separation was achieved using an Eclipse XDB-C18 column (50 $\mathrm{mm} \times$ $2.1 \mathrm{~mm}$ i.d., particle size $3.5 \mu \mathrm{m}$; Agilent Technologies, Singapore) using gradient elution with mobile phases $0.1 \%$ formic acid in water and acetonitrile, at a flow rate of $0.3 \mathrm{~mL} / \mathrm{min}$. This was coupled to a Shimadzu LCMS8030 mass spectrometer for analyte identification and quantification.
Fig. 1 Order of events during experimental sessions. VAS visual analog scale, STAI State Trait Anxiety Inventory, DSST Digit Symbol Substitution Task, DAT Divided Attention Task, PASAT Paced Auditory Serial Addition Task, ADSES Adelaide Driving Self-Efficacy Scale

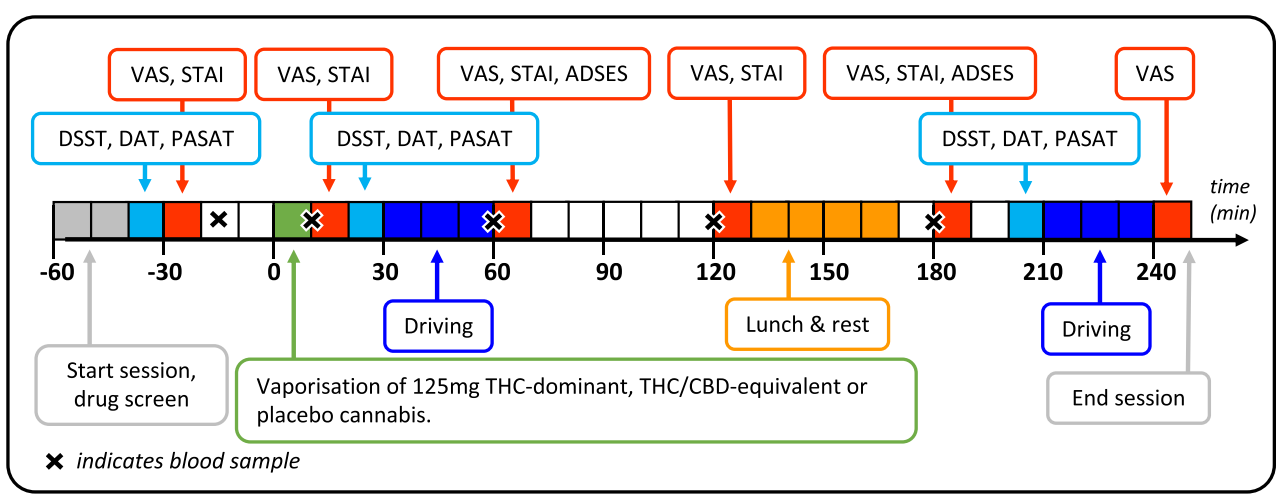




\section{Driving simulator}

The custom-built driving simulator (Hyperdrive, Adelaide, Australia) consisted of a fixed-base equipped with original vehicle controls (steering wheel, indicators, seat, safety belt), hi-resolution Fanatec ${ }^{\circledR}$ pedals, and a servo motor wheel base (Endor AG, Landshut, Germany) linked to four networked computers running SCANeR ${ }^{\mathrm{TM}}$ Studio simulation engine (v1.6, OKTAL, Paris, France). Visual images were displayed on three 32-in. LCD monitors using three channels set to provide a $100^{\circ}$ field of view. A digital dashboard displayed speed, rpm, and status of vehicular control systems (e.g., traction control). A complete rear visual scene was displayed on three separate channels (rear vision mirror, left, and right-side mirrors). Graphics refreshed at a rate of $60 \mathrm{~Hz}$, and data were sampled at a rate of $20 \mathrm{~Hz}$. Surround sound provided auditory feedback, and force feedback steering provided haptic feedback. Data collected by the simulator's software program included measures of lateral control (lateral position, steering wheel angle), longitudinal control (speed, acceleration), and interaction with other vehicles.

\section{Driving scenarios}

The driving simulation started with a 5-min highway carfollowing task in which participants were required to follow and maintain a constant distance (headway) to a lead vehicle that would accelerate or decelerate every $30 \mathrm{~s}$ in a sinusoidal manner (between 90 and $110 \mathrm{~km} / \mathrm{h}$ ). The task occurred on a two-lane, dual-carriageway highway in steady highway traffic. Outcome measures included standard deviation of lateral position (SDLP; a measure of lane weaving (Verster and Roth 2011)), mean headway (i.e., distance to the lead vehicle), and standard deviation of headway.

The remainder of the task (25 min; "secondary driving task") consisted of highway and rural segments. Participants were instructed to follow the spoken GPS directions and drive as they normally would. Highway segments involved a two-lane, dual-carriageway road with posted $110 \mathrm{~km} / \mathrm{h}$ speed limits, rural segments involved winding single-lane roads with various posted speed signs $(60-100 \mathrm{~km} / \mathrm{h})$, and intersections with and without signalcontrols. Outcome measures included SDLP, mean speed (MSP), and standard deviation of speed (SDSP). Throughout the task, there were various hazards (e.g., roadworks, aggressive drivers), cyclists, pedestrians, and traffic in variable density. To minimize familiarity, the appearance (i.e., make, model and color) of other vehicles was generated randomly for each drive. The time of day for each drive was set to match the actual time of testing. Tests of simulated driving began $30 \mathrm{~min}$ (T1) and $210 \mathrm{~min}$ (T2) after dosing.

\section{Cognitive tasks}

Cognitive/psychomotor performance was assessed using three computerized tasks which are known to be sensitive to the impairing effects of THC (Vandrey et al. 2017). These included the Digit Symbol Substitution Task (DSST; (Mcleod et al. 1982)), Divided Attention Task (DAT; (Kleykamp et al. 2010)), and Paced Auditory Serial Addition Task (PASAT; (Herrmann et al. 2015)). Performance assessments were completed in this order at baseline and $20 \mathrm{~min}$ (T1) and $200 \mathrm{~min}$ (T2) after dosing.

In the DSST, participants were presented with a series of geometric patterns labeled from 1 to 9 , each consisting of an array of filled and blank squares in a $3 \times 3$ grid. When a number appeared in the middle of the screen, participants were instructed to replicate the pattern corresponding to that array using the numeric keypad of a computer keyboard. Participants had $90 \mathrm{~s}$ to replicate as many patterns as possible. Outcome measures included number of patterns correct and accuracy (number of patterns correct/number of patterns attempted).

In the DAT, participants were required to track a horizontally moving stimulus on the screen using their mouse while simultaneously responding to peripheral visual stimuli by clicking the left mouse button whenever a number in any corner of the screen matched a target number presented at the bottom of the screen. Outcome measures included mean distance of the cursor from the target (tracking error), the number of target numbers correctly identified (/24), and response time.

In the PASAT, participants watched single digits appear on the screen and were instructed to sum each new digit with the preceding one. Participants responded by clicking on the correct answer from a list of numbers (1-10) presented on the screen. Outcome measures included response time on correct trials and the total number of correct trials (/90).

\section{Subjective drug effects}

Subjective drug effects were assessed at baseline and 15, 60, 120, 180, and 240 min after dosing using a series of Visual Analog Scales (VAS). Participants rated on a $100-\mathrm{mm}$ line their responses to the statements: "Strength of drug effect", "Liking of drug effect", "Stoned," "Sedated," "Anxious," and "Confident to drive". All scales were unipolar except for "Liking of drug effect" which was bipolar (dislike very much - like very much). The State-Trait Anxiety Inventory (Y-1 - state form only) (Spielberger 1983) was also administered at baseline and 15, 60,120 and 180 min after dosing. Self-reported driving ability was further assessed by the Adelaide Driving Self-Efficacy Scale (George et al. 2007) (which provides a score from 0 to 120 ) at baseline and at 60 and $180 \mathrm{~min}$ after dosing. 


\section{Statistical analysis}

Sample size was determined by a power calculation based on the effect size $\left(\eta p^{2}=0.14\right)$ associated with the main effect of Dronabinol on simulated driving performance reported in a previous study (Veldstra et al. 2015). Data from the driving simulator tasks were reviewed and cleaned to remove recognizable artifacts (e.g., increased SDLP while overtaking other vehicles). All data were analyzed in SPSS v24 (IBM Corp, Armonk, NY) with Linear Mixed Models (LMMs). The restricted maximum likelihood method was selected, and a firstorder autoregressive (AR1) covariance structure was specified for repeated factors as it provided the lowest Akaike information criterion (AICC) model fit values. Fixed factors included treatment ( 3 levels), time (2, 3, and 5 levels for driving, cognitive and pharmacokinetic/subjective drug effects data, respectively), session (3 levels), and treatment by time. For data which included baseline assessment (i.e., cognitive, pharmacokinetic, and subjective drug effect data), baseline scores were included in the model as a covariate. In each model, planned Bonferroni pairwise comparisons were used to compare treatment means at each level of time. For additional pharmacokinetic data (e.g., area under the curve), nonparametric Wilcoxon signed-rank tests were used to assess differences between treatments. The statistical significance level was set at $p<0.05$.

\section{Results}

\section{Participants}

Table 1 presents the characteristics of the 14 healthy adults (11 males, 21-38 years old) who completed all three test sessions between December 2017 and April 2018. Three additional

Table 1 Characteristics of participants

\begin{tabular}{ll}
\hline Number of participants & 14 \\
\hline Sex (male/female) & $11 / 3$ \\
Race (White/Asian) & $13 / 1$ \\
Age (years) & $27.5(4.5)$ \\
BMI (kg/m ${ }^{2}$ ) & $25.5(4.9)$ \\
Alcohol intake frequency (no. of days per month) & $7.1(5.3)$ \\
Age of first cannabis use (years) & $15.9(2.6)$ \\
Days used cannabis in last 28 days (no.) & $4.5(4.8)$ \\
Days used cannabis use in last 3 months (no.) & $11.2(8)$ \\
Years of driving experience (no.) & $9.6(4.1)$ \\
Total days driven in last 28 days (no.) & $11.9(10.6)$ \\
Typical wait before driving after consuming cannabis (h) & $5.9(7)$ \\
\hline
\end{tabular}

Data are shown as means (SD) or as frequency. $B M I$ body mass index participants began, but did not complete, the study: one withdrew after the first session, another was discharged for protocol non-compliance, and another was discharged prior to drug administration following multiple elevated baseline blood pressure and heart rate readings. None of the 14 participants reported regular use of any medications, and all participants provided negative oral fluid drug tests on the morning of each test session. Blinding was assessed at the end of the trial by asking participants which cannabis type they thought they received in each of the three sessions. All 14 participants correctly identified the placebo session, and it was commonly reported that less vapor was produced by the placebo cannabis.

\section{Subjective measures}

As shown in Fig. 2, there was a significant main effect of treatment on "Stoned" $[F(2,43.27)=64.25, p<0.001]$, "Strength of drug effect $[\mathrm{F}(2,45.81)=70.24, p<.001]$, "Sedated" $[F(2,59.18)=15.25, p<.001]$ and "Liking of drug effect" $[F(2,49.43)=17.53, p<0.001]$. Subjective ratings did not differ between the THC and THC/CBD conditions at any point in time, however only THC/CBD increased ratings of "Sedated" when compared with placebo at $240 \min (p=$ $0.008)$. Treatment also significantly affected ratings of "Anxious" $[F(2,44.52)=5.40, p=0.008]$ (Fig. 2) and STAI scores $[F(2,51.08)=7.05, p=0.002]$. On both measures, both THC and THC/CBD produced a modest but significant increase in ratings of "Anxious" at 15 min when compared with placebo, but only THC did so at $1 \mathrm{~h}$. Finally, treatment significantly affected ratings of "Confident to drive" $[\mathrm{F}(2,53.52)=$ 27.68, $p<.001$ ] (Fig. 2) and Adelaide Driving Self-Efficacy Scale scores $[\mathrm{F}(2,108.417)=20.41, p<.001]$ (Fig. S1) such that both $\mathrm{THC}$ and $\mathrm{THC} / \mathrm{CBD}$ significantly decreased scores on both measures when compared with placebo up to $240 \mathrm{~min}$ after vaporizing.

\section{Driving performance}

Mean (SD) values of driving outcome measures are presented in Table 2.

Car-following task In the car-following section of the driving task, there was a significant main effect of treatment on SDLP $[\mathrm{F}(2,31.43)=11.44, p<0.001]$ but no treatment by time interaction. When compared with placebo, THC increased SDLP by $3.91 \mathrm{~cm}(p=0.019)$ and $3.84 \mathrm{~cm}(p=0.041)$ at T1 and T2, respectively, while THC/CBD increased SDLP by $4.28 \mathrm{~cm}$ $(p=0.036)$ and $5.28 \mathrm{~cm}(p=0.003)$. Mean headway (i.e. distance to the lead vehicle) did not differ between conditions $[\mathrm{F}(2,30.17)=2.73, p=0.081]$, however standard deviation of headway $\operatorname{did}[\mathrm{F}(2,27.743)=4.96, p=0.014]$. When 
Fig. 2 Mean (SEM) participant ratings of "Stoned", "Strength of drug effect", "Sedated", "Liking of drug effect", "Anxious" and "Confident to drive" assessed using $1-100 \mathrm{~mm}$ visual analog scales after vaporization of placebo, THC-dominant, and THC/ CBD-equivalent cannabis. All scales were unipolar except for "Liking of drug effect". $B L$ baseline
"Stoned"
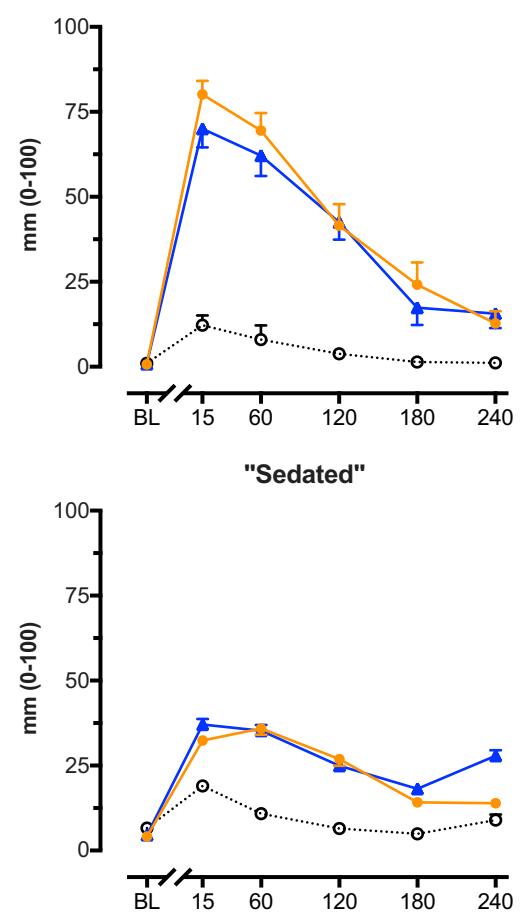

"Anxious"

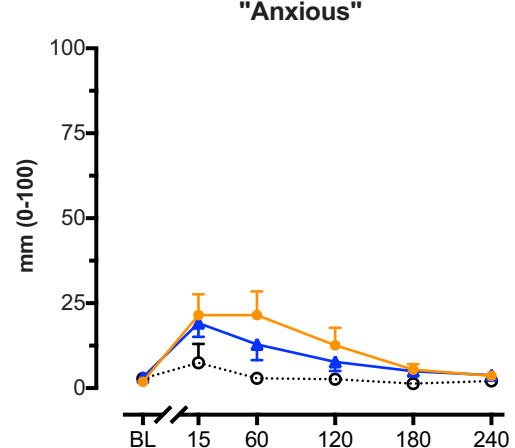

"Strength of drug effect"

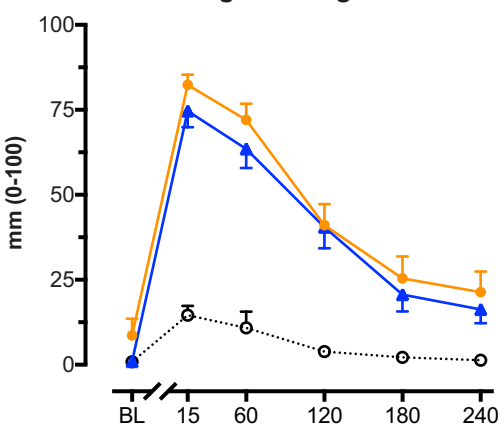

"Liking of drug effect"

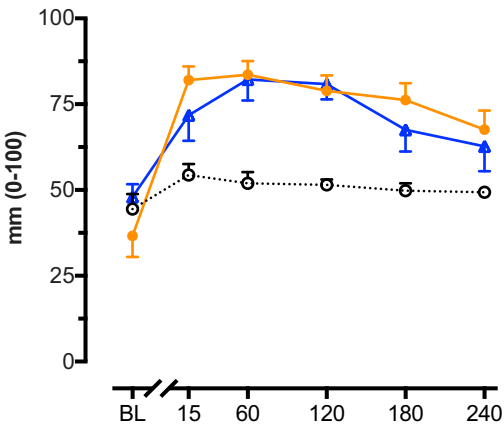

"Confident to drive"

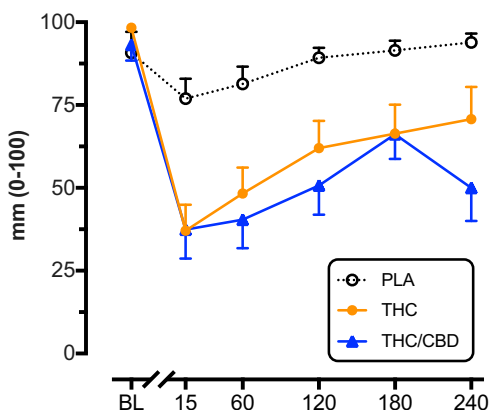

Time since vaporization (min)

Table 2 Driving performance [means (SD)] at T1 and T2 (30 min and $210 \mathrm{~min}$ ) after vaporization of placebo, THC-dominant, and THC/CBDequivalent cannabis

\begin{tabular}{|c|c|c|c|c|c|c|}
\hline & \multicolumn{3}{|l|}{$\mathrm{T} 1$} & \multicolumn{3}{|l|}{$\mathrm{T} 2$} \\
\hline & Placebo & THC & $\mathrm{THC} / \mathrm{CBD}$ & Placebo & THC & $\mathrm{THC} / \mathrm{CBD}$ \\
\hline \multicolumn{7}{|l|}{ Car-following task } \\
\hline $\operatorname{SDLP}(\mathrm{cm})$ & $19.29(6.47)$ & $23.21(4.77)^{*}$ & $23.50(7.36)^{*}$ & $19.00(5.11)$ & $22.86(6.00)^{*}$ & $24.21(6.39)^{* *}$ \\
\hline Headway (m) & $75.38(23.32)$ & $85.28(30.48)$ & $86.01(41.48)$ & $71.82(23.81)$ & $82.91(27.04)$ & $90.13(36.59)$ \\
\hline SD headway (m) & $29.10(11.92)$ & $30.82(12.97)$ & $40.59(25.97)$ & $28.22(13.50)$ & 34.33 (14.67) & $42.79(24.75)^{*}$ \\
\hline \multicolumn{7}{|c|}{ Secondary driving task } \\
\hline $\operatorname{SDLP}(\mathrm{cm})$ & $27.07(5.41)$ & $28.43(5.52)$ & $28.5(6.62)$ & $28.43(6.31)$ & $28.36(5.79)$ & $28.71(6.34)$ \\
\hline Speed $(\mathrm{km} / \mathrm{h})$ & $82.16(4.02)$ & $81.47(3.17)$ & $82.72(3.48)$ & $84.21(3.11)$ & $83.72(3.56)$ & $83.46(3.41)$ \\
\hline $\operatorname{SDSP}(\mathrm{km} / \mathrm{h})$ & $26.96(3.46)$ & $27.28(2.19)$ & $25.78(1.88)$ & $26.91(2.34)$ & $26.30(2.17)$ & $26.45(1.57)$ \\
\hline
\end{tabular}

$S D L P$ standard deviation of lateral position, $S D$ distance standard deviation of distance, SDSP standard deviation of speed. Bonferroni post hoc tests were used to compare treatment means at each level of time. ${ }^{*}$ Indicates significantly different from placebo $(p<0.05) .{ }^{* * *}$ Indicates significantly different from placebo $(p<0.01)$ 
compared with placebo, the THC/CBD condition had greater standard deviation of headway at T2 by $15.33 \mathrm{~m}(p=0.044)$.

Secondary driving task In the secondary driving task, treatment did not significantly affect lateral or longitudinal vehicular control parameters including SDLP, MSP and SDSP. Separate analyses of SDLP over different segments (i.e. highway and rural) of the drive indicated that cannabis did not significantly impair driving at any point during the $25 \mathrm{~min}$ task.

\section{Cognitive task performance}

Mean (SD) values of cognitive task performance measures are presented in Table 3.

DSST In the DSST, there was a trend towards a main effect of treatment on accuracy $[F(2,50.52)=2.80, p=0.07]$ and number of items correct $[F(2,49)=2.55, p=0.09]$. Planned Bonferroni comparisons showed that participants exhibited fewer correct items at $\mathrm{T} 1$ in the THC condition when compared with placebo $(p=0.017)$ (Fig. 3). There were no significant differences between conditions at $\mathrm{T} 2$.

DAT There was a significant effect of treatment on tracking error $[F(2,54.73)=5.52, p=0.007]$, which was increased at $\mathrm{T} 1$ in the THC/CBD condition when compared with both the placebo $(p<0.001)$ and THC $(p=0.042)$ conditions (Fig. 3). There were no significant effects on response time or number of items correct and no differences between treatments at $\mathrm{T} 2$.

PASAT In the PASAT there was a significant effect of treatment on both number of correct trials $[F(2,52.52)=6.30, p=0.004]$ and response time $[F(2,52.93)=11.09, p<0.001]$ (Fig. 3). Fewer correct trials $(p<0.001)$ and increased response time $(p<0.001)$ were observed at T1 in the THC/CBD condition compared with placebo. The THC condition significantly increased response time $(p<0.001)$, but not differences in number of correct trials at T1 compared with placebo (Fig. 3). There were no significant differences between conditions at $\mathrm{T} 2$.

\section{Plasma cannabinoid concentrations}

Plasma concentrations of THC, its secondary metabolite (11$\mathrm{OH}-\mathrm{THC}$ ), terminal metabolite (THC-COOH), and CBD, are shown in Fig. 4. Analysis revealed a significant main effect of treatment on plasma THC $[F(2,96.94)=83.35, p<0.001], 11$ $\mathrm{OH}-\mathrm{THC}[F(2,93.86)=31.37, p<0.001]$, THC-COOH $[F(2,136.64)=93.35, p<0.001]$, and $\operatorname{CBD}[F(2,107.87)=$ $136.61, p<0.001]$. The main effect of time and the treatment by time interaction was also highly significant in all three

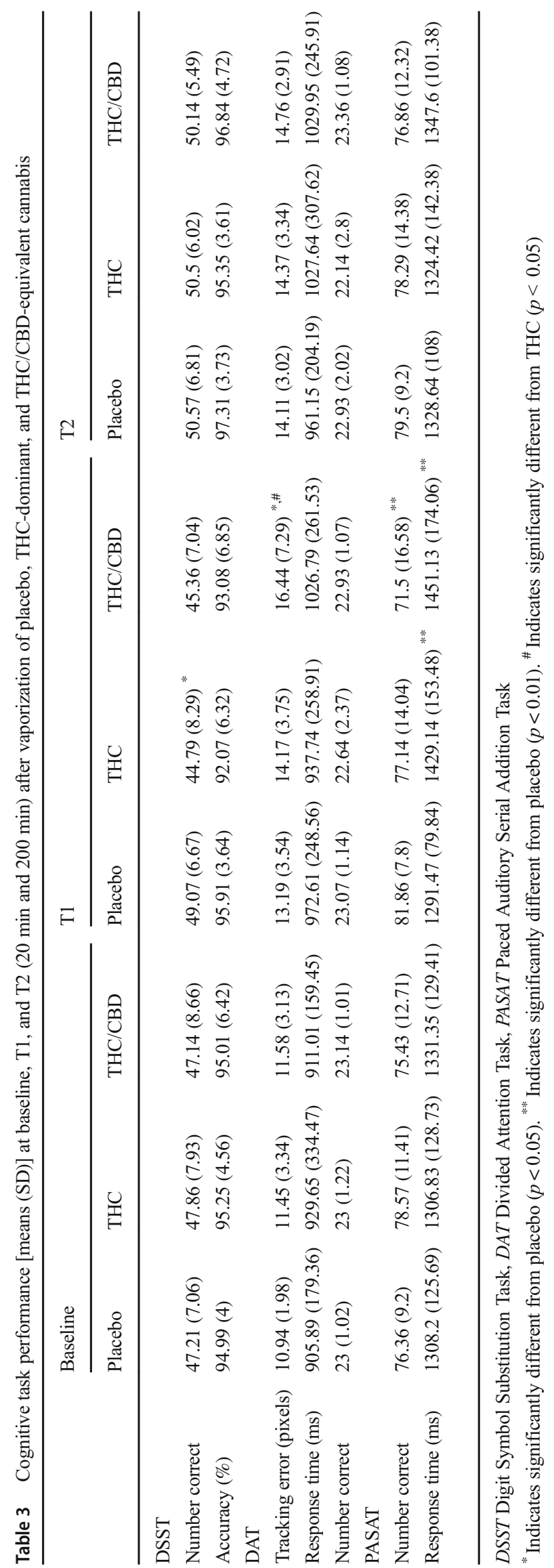


Fig. 3 Mean (SEM) performance on the Digit Symbol Substitution Task (DSST), Divided Attention Task (DAT), and Paced Auditory Serial Addition Task (PASAT) after vaporization of placebo, THC-dominant, and THC/CBDequivalent cannabis. ${ }^{*} p<0.05$, $* * p<0.01, * * * p<0.001$, Bonferroni post-hoc. $B L$ baseline
Digit Symbol Substitution Task: Number of Items Correct

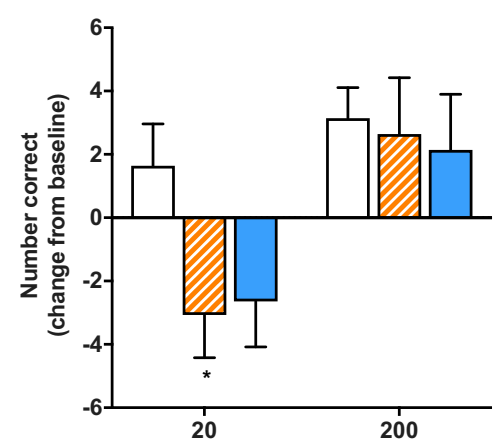

Paced Auditory Serial Addition Task: Number of Correct Trials

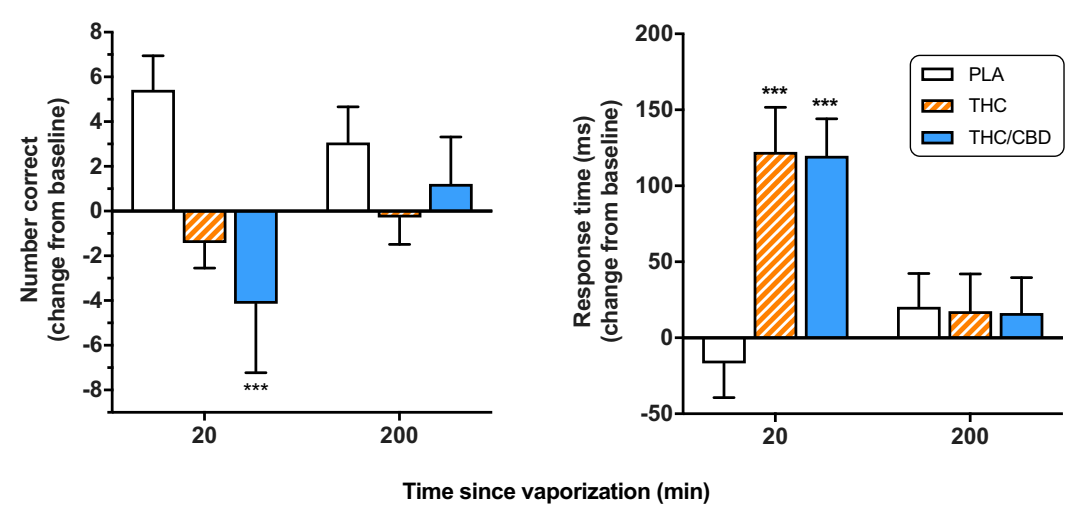

Divided Attention Task: Tracking Error

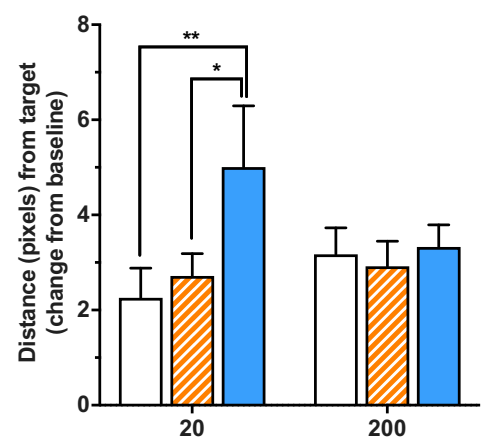

Paced Auditory Serial Addition Task: Response Time models. When compared with the THC condition, the THC/ CBD condition displayed increased peak plasma concentrations $\left(C_{\max }\right)$ by an additional $8.60 \mathrm{ng} / \mathrm{mL}(p<0.001)$. Mean 11-OH-THC $C_{\max }$ was slightly higher in the THC/CBD condition and $\mathrm{THC}-\mathrm{COOH} C_{\max }$ marginally lower, although these differences were not significant. The area under the curve $\left(\mathrm{AUC}_{0->3 \mathrm{~h}}\right)$ for THC was higher in the THC/CBD condition $(51.37 \mathrm{ng} / \mathrm{mL} \times \mathrm{h})$ than in the THC condition $(42.51 \mathrm{ng} / \mathrm{mL} \times \mathrm{h})$, although this difference did not reach significance $(p=0.064)$. 11-OH-THC $\mathrm{AUC}_{0->3} \mathrm{~h}$ was 3.29 (THC) and $4.00(\mathrm{THC} / \mathrm{CBD}) \mathrm{ng} / \mathrm{mL} \times \mathrm{h}$. THC-COOH $\mathrm{AUC}_{0->3} \mathrm{~h}$ was 49.75 (THC) and (THC/CBD) $49.30 \mathrm{ng} /$ $\mathrm{mL} \times \mathrm{h}$. Mean $T_{\max }$ was calculated as $0.17 \mathrm{~h}$ for all analytes.

\section{Discussion}

Overall, the results of the current study indicate that THC/CBDequivalent and THC-dominant cannabis produce similar subjective effects and impairment. Vaporization of either chemovar significantly impaired driving during a car-following task and reduced confidence in driving up to $4 \mathrm{~h}$ later. Cognitive function was also impaired; however, these effects were more transient and had largely dissipated at the later time point.
Pharmacokinetic data showed that peak plasma concentrations of THC appeared higher when CBD was co-administered.

The finding that CBD in cannabis does not greatly alter the subjective effects of THC is consistent with the findings of several previous studies (Hindocha et al. 2015; Morgan et al. 2010a; Roser et al. 2008; Juckel et al. 2007). Both active cannabis types significantly increased ratings of subjective drug effects (e.g., "stoned," "strength of drug effect") relative to placebo. These effects were maximal at $15 \mathrm{~min}$ and declined steadily thereafter. Participants also expressed markedly reduced confidence in their driving ability immediately after vaporization and even $240 \mathrm{~min}$ later. There were only subtle differences between the effects of the two active drug conditions in ratings of "Sedated" or "Anxious," with THC/CBD equivalent cannabis appearing to cause slightly greater sedation at $240 \mathrm{~min}$ and slightly less anxiety at $60 \mathrm{~min}$ compared with THC-dominant cannabis.

Both active cannabis types significantly increased SDLP (i.e., lane weaving) during the car-following task at $30 \mathrm{~min}$ and $210 \mathrm{~min}$ after vaporizing. Although SDLP may not directly predict crash risk, it is a highly sensitive index of THC and alcohol-impaired driving (Veldstra et al. 2015; Bosker et al. 2012; Downey et al. 2013; Hartman et al. 2015; Irwin et al. 2017) and is a widely used proxy for driver safety (Verster and 
Fig. 4 Mean (SEM) plasma concentrations $(\mathrm{ng} / \mathrm{mL})$ of $\mathrm{THC}$, $\mathrm{CBD}, 11-\mathrm{OH}-\mathrm{THC}$, and THC$\mathrm{COOH}$ after vaporization of placebo, THC-dominant, and THC/ CBD-equivalent cannabis
THC

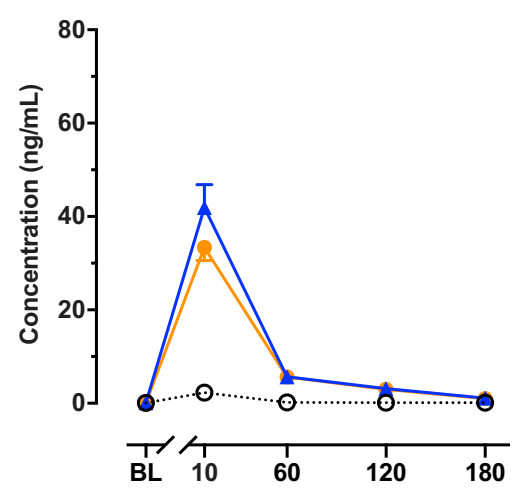

11-OH-THC

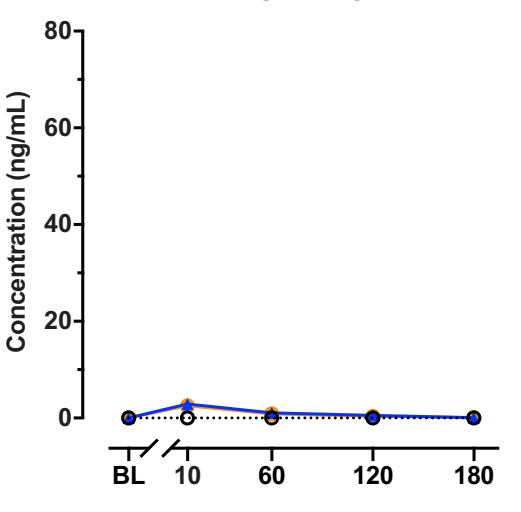

CBD
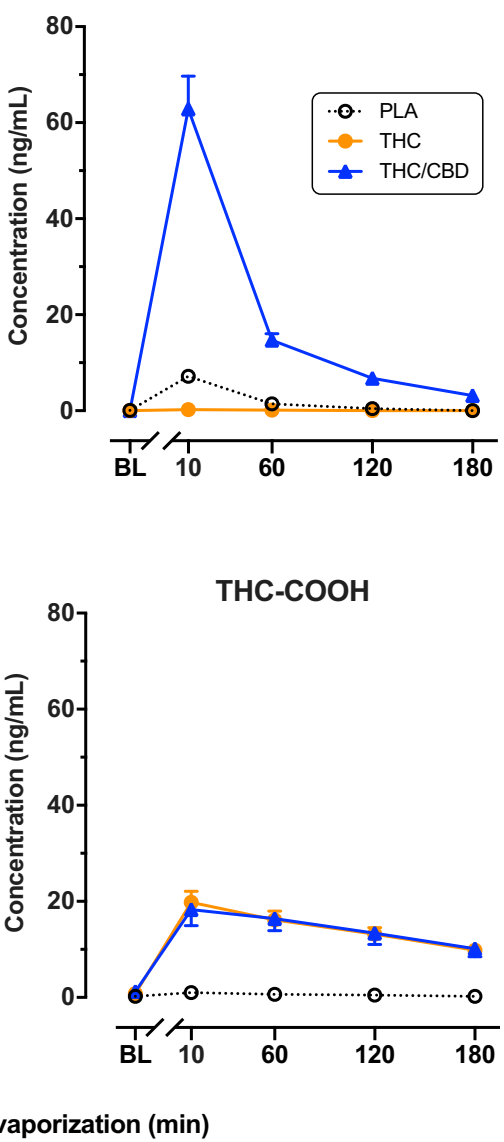

Roth 2011). At the 30-min time point, both the THC and THC/ CBD treatments increased SDLP by approximately $4 \mathrm{~cm}$, comparable to the effects of $20 \mathrm{mg}$ dronabinol (Bosker et al. 2012), $19 \mathrm{mg}$ smoked THC (Lenné et al. 2010), and 200- $\mu \mathrm{g} / \mathrm{kg}$ smoked THC (equivalent to $15 \mathrm{mg}$ THC for a $75 \mathrm{~kg}$ person) (Ramaekers et al. 2000). Participants in both active treatment conditions also tended to leave a larger and more variable gap between them and the lead vehicle, which may indicate an attempt to compensate for perceived impairment (Hartman et al. 2016).

In the secondary driving task, however, neither lateral (SDLP) nor longitudinal (mean speed, SDSP) control measures were affected by cannabis. While the car-following task required participants to constantly monitor and adapt to the speed changes of a lead vehicle while driving in steady highway traffic, the secondary driving task was relatively simple. Although there were some hazards requiring driver interaction (e.g., roadworks), the drive was monotonous and involved long stretches of single-lane rural roads with low traffic density. It is possible that this task underestimated the complexities of realworld driving, although such situations are far from uncommon when driving in rural areas. A recent study similarly found that $13 \mathrm{mg}$ THC (100 mg cannabis containing 12.9\% THC) administered via vaporization did not affect simple driving-related task performance despite reducing confidence in driving ability and performance on complex cognitive tasks (Ogourtsova et al. 2018). The impairing effects of THC are known to increase with task complexity (Hartman and Huestis 2013) and therefore may only be evident under demanding driving conditions.

Cannabis administration produced acute cognitive impairment in the DSST, PASAT, and DAT tasks employed in the current study. This agrees with results of a very recent study (Spindle et al. 2018) in which vaporized cannabis (10 mg THC) produced moderate but significant impairment on outcomes in these same tasks. The modest impairment in the DSST involved a significant decrease in correct responses in the THC treatment condition. In the DAT, response time to peripheral stimuli was largely unaffected by cannabis, while tracking error - a measure sensitive to both THC (Verster and Roth 2011; Ramaekers et al. 2009) and alcohol (Jongen et al. 2016) - was increased considerably in the THC/CBD condition relative to the other two conditions. The PASAT was most sensitive to cannabis effects, with processing speed significantly impaired in both the THC and THC/CBD conditions, and number of correct trials significantly decreased in the THC/CBD condition. Collectively, these results suggest that the use of THC/CBDequivalent cannabis does not prevent the cognitive impairment seen with THC-dominant cannabis and may, under some circumstances, cause greater impairment. 
Curiously, at $210 \mathrm{~min}$ following vaporization of either THC or THC/CBD, participants still showed impaired lateral vehicular control (i.e., increased SDLP) and reported reduced confidence in driving ability despite an apparent resolution of cognitive impairment and a dissipation of subjective drug effects (e.g. "stoned"). It is thus possible that these cognitive tests (DSST, DAT, PASAT) are only sensitive to the impairing effects of cannabis when the magnitude of effect is large and unambiguous (i.e., during acute intoxication). This may be because these tests predominantly assessed controlled and conscious processes (e.g., response time, attention) that are more accessible to compensatory mechanisms than road tracking (lateral control), which is a highly automated and learned skill that is particularly vulnerable to internal disruptions (e.g., CNS drug effects) (Robbe 1998). It is interesting to note that participants could accurately assess their driving impairment even in the absence of salient subjective drug effects, and that THC-induced driving impairment may persist well beyond the period of acute intoxication. This observation may have implications for the advice given to medicinal cannabis patients around driving safety.

Analysis of plasma cannabinoid concentrations showed that peak THC levels were significantly higher in the THC/ CBD condition than in the THC condition. CBD inhibits certain forms of drug metabolism (Stout and Cimino 2014) including those involving the CYP3A4, CYP2C9, and CYP2C19 isoforms involved in THC metabolism (Yamaori et al. 2011). It is conceivable that such inhibition could lead to increased plasma THC concentrations. Two previous studies found no evidence of a pharmacokinetic interaction between THC and CBD with buccal or oral administration in humans (Karschner et al. 2011; Nadulski et al. 2005); however, the maximum CBD concentrations obtained in these studies were an order of magnitude lower than those obtained here with vaporized cannabis. It should also be acknowledged, as an alternative explanation of these results, that THC/CBD equivalent vapor may have a different sensory quality to THCdominant vapor and that this might lead to subtle differences in dose titration and self-administration, and hence higher plasma THC concentrations with THC/CBD equivalent cannabis. As inhalation remains the most widely used method of both recreational and medicinal cannabis administration, these preliminary findings warrant further investigation and verification.

\section{Strengths and limitations}

Major strengths of this study include a rigorous double-blind, placebo-controlled, within-subjects, and crossover design; the use of the Mighty Medic vaporizer, an approved medical device for cannabis administration in both Canada and the EU; assessment of driving and cognitive function at various timepoints following vaporization; and repeated sampling of blood.
Limitations include the absence of a CBD-only condition: this was omitted because vaporization of $\mathrm{CBD}$ alone is uncommon in the real world and because acute administration of $\mathrm{CBD}$ in previous human laboratory studies has not produced notable drug effects that are suggestive of intoxication or impairment (Haney et al. 2016; Martin-Santos et al. 2012; Winton-Brown et al. 2011; Borgwardt et al. 2008; Babalonis et al. 2017; Dalton et al. 1976; Hollister 1973). It is also acknowledged that the study used a THC dose sufficient to produce robust subjective and behavioral effects in infrequent cannabis users, but that regular cannabis users may use considerably higher doses than used here. Future studies should therefore consider using multiple THC doses and higher THC and CBD ratios (e.g., 1:10) than the 1:1 ratio that we examined here. Another limitation of this study is the relatively small sample size. The window for participant recruitment was limited by expiration of the study drug and regulatory process in Australia which made further study drug importation difficult. Nonetheless, a range of highly significant results were obtained.

This study was also limited to healthy volunteers who were only occasional cannabis users. Cannabis use history (and therefore tolerance) strongly affects individual responses to THC, with occasional users being significantly more susceptible to impairment than regular users (Bosker et al. 2012; Ramaekers et al. 2009; Desrosiers et al. 2015). Regular cannabis users (i.e., daily or near daily) may therefore be less impaired in the experimental paradigms used here. We also note that while there are obvious advantages to using a driving simulator (i.e., greater experimental control and minimal risk), the complexities of real-world driving may not be replicated entirely. A carefully controlled on-road driving study would therefore be useful in verifying the results obtained here. Further research is also needed to validate the cognitive tasks used here among others as predictors of real-world driving performance.

Finally, the fact that all 14 participants correctly identified the placebo session suggests an issue with placebo cannabis preparations that is difficult to resolve, particularly when it is administered alongside active cannabis in a within-subjects crossover design. Of course, blinding is always a challenge in psychopharmacological research when an active treatment has distinctive and salient psychoactive effects. Placebo cannabis that retains some of the aroma and taste of active cannabis may still be preferable relative to alternatives such as inert herbal mixtures.

\section{Conclusion}

In conclusion, this study indicates that vaporized cannabis with equivalent concentrations of THC and CBD causes similar impairment of driving and cognition to THC-dominant 
cannabis, and does not produced substantially different subjective effects. In fact, the presence of CBD may increase plasma concentrations of THC, and subtly increase some measures of cognitive and driving impairment. These results have significant implications for individuals using medicinal and recreational cannabis containing both THC and CBD.

Acknowledgements We thank Tilray for supplying the cannabis to conduct this study, and Storz and Bickel for supplying the Mighty Medic vaporizers. We thank Dr. Hamish MacDougall, Ms. Zara Mills, Ms. Consuelo Rivas, and Dr. Jordyn Stuart for their invaluable contributions and we wish to sincerely acknowledge the support and contributions of the late Associate Professor David Allsop.

Funding This study was supported by the Lambert Initiative for Cannabinoid Therapeutics, a philanthropically-funded center for medicinal cannabis research at the University of Sydney.

\section{Compliance with ethical standards}

Informed consent All participants gave written informed consent and all procedures were approved by the Sydney Local Health District (RPAH Zone) Human Research Ethics Committee.

Conflict of interest Nicholas Lintzeris has received funding for sponsored research studies from Camurus and has provided consultancies for Indivior and Mundipharma for unrelated work. Ryan Vandrey has received consulting fees from Zynerba Pharmaceuticals, Battelle Memorial Institute, and Canopy Health Innovations Inc. and has received compensation for being on the advisory boards for Insys Therapeutics, Brain Solutions Inc., and The Realm of Caring Foundation. Paul Haber has received research funding from Camurus, and has provided consultancies for Indivior, Abbvie, and Gilead for unrelated work. Iain McGregor acts as a consultant to Kinoxis Therapeutics and is an inventor on several patents relating to novel cannabinoid therapeutics. The other authors have no conflicts of interest to disclose.

Open Access This article is distributed under the terms of the Creative Commons Attribution 4.0 International License (http:// creativecommons.org/licenses/by/4.0/), which permits unrestricted use, distribution, and reproduction in any medium, provided you give appropriate credit to the original author(s) and the source, provide a link to the Creative Commons license, and indicate if changes were made.

\section{References}

Babalonis S, Haney M, Malcolm RJ et al (2017) Oral cannabidiol does not produce a signal for abuse liability in frequent marijuana smokers. Drug Alcohol Depend 172:9-13

Bhattacharyya S, Morrison PD, Fusar-Poli P et al (2010) Opposite effects of delta-9-tetrahydrocannabinol and cannabidiol on human brain function and psychopathology. Neuropsychopharmacology 35:764-774

Boggs DL, Nguyen JD, Morgenson D et al (2018) Clinical and preclinical evidence for functional interactions of Cannabidiol and Delta(9)tetrahydrocannabinol. Neuropsychopharmacology 43:142-154

Borgwardt SJ, Allen P, Bhattacharyya S et al (2008) Neural basis of $\Delta-9$ tetrahydrocannabinol and cannabidiol: effects during response inhibition. Biol Psychiatry 64:966-973

Bosker WM, Kuypers KP, Theunissen EL et al (2012) Medicinal $\Delta 9$ tetrahydrocannabinol (dronabinol) impairs on-the-road driving performance of occasional and heavy cannabis users but is not detected in standard field sobriety tests. Addiction 107:1837-1844

Capler R, Bilsker D, Van Pelt K et al. (2017) Cannabis use and driving: evidence review. (Accessed 12 Sept 2018)

Cuba LF, Salum FG, Cherubini K et al (2017) Cannabidiol: an alternative therapeutic agent for oral mucositis? J Clin Pharm Ther 42:245-250

Dalton WS, Martz R, Lemberger L et al (1976) Influence of cannabidiol on delta-9-tetrahydrocannabinol effects. Clin Pharmacol Ther 19: 300-309

Desrosiers NA, Ramaekers JG, Chauchard E et al (2015) Smoked cannabis' psychomotor and neurocognitive effects in occasional and frequent smokers. J Anal Toxicol 39:251-261

Downey LA, King R, Papafotiou K et al (2013) The effects of cannabis and alcohol on simulated driving: influences of dose and experience. Accid Anal Prev 50:879-886

George S, Clark M, Crotty M (2007) Development of the Adelaide driving self-efficacy scale. Clin Rehabil 21:56-61

Haney M, Malcolm RJ, Babalonis S et al (2016) Oral cannabidiol does not alter the subjective, reinforcing or cardiovascular effects of smoked cannabis. Neuropsychopharmacology 41:1974-1982

Hartman RL, Huestis MA (2013) Cannabis effects on driving skills. Clin Chem 59:478-492

Hartman RL, Brown TL, Milavetz G et al (2015) Cannabis effects on driving lateral control with and without alcohol. Drug Alcohol Depend 154:2537

Hartman RL, Brown TL, Milavetz G et al (2016) Cannabis effects on driving longitudinal control with and without alcohol. J Appl Toxicol 36:14181429

Herrmann ES, Cone EJ, Mitchell JM et al (2015) Non-smoker exposure to secondhand cannabis smoke II: effect of room ventilation on the physiological, subjective, and behavioral/cognitive effects. Drug Alcohol Depend 151:194-202

Hindocha C, Freeman TP, Schafer G et al (2015) Acute effects of delta-9tetrahydrocannabinol, cannabidiol and their combination on facial emotion recognition: a randomised, double-blind, placebo-controlled study in cannabis users. Eur Neuropsychopharmacol 25: 325-334

Hollister L (1973) Cannabidiol and cannabinol in man. Experientia 29: $825-826$

Hollister LE, Gillespie H (1975) Interactions in man of delta-9-tetrahydrocannabinol. II. Cannabinol and cannabidiol. Clin Pharmacol Ther 18:80-83

Ilan AB, Gevins A, Coleman M et al (2005) Neurophysiological and subjective profile of marijuana with varying concentrations of cannabinoids. Behav Pharmacol 16:487-496

Irwin C, Iudakhina E, Desbrow B et al (2017) Effects of acute alcohol consumption on measures of simulated driving: a systematic review and meta-analysis. Accid Anal Prev 102:248-266

Jongen S, Vuurman EF, Ramaekers JG et al (2016) The sensitivity of laboratory tests assessing driving related skills to dose-related impairment of alcohol: a literature review. Accid Anal Prev 89:31-48

Juckel G, Roser P, Nadulski $T$ et al (2007) Acute effects of $\Delta$ 9-tetrahydrocannabinol and standardized cannabis extract on the auditory evoked mismatch negativity. Schizophr Res 97:109-117

Karschner EL, Darwin WD, Goodwin RS et al (2011) Plasma cannabinoid pharmacokinetics following controlled oral delta9-tetrahydrocannabinol and oromucosal cannabis extract administration. Clin Chem 57:66-75

Kevin RC, Allsop DJ, Lintzeris N et al (2017) Urinary cannabinoid levels during nabiximols (Sativex ${ }^{\circledR}$ )-medicated inpatient cannabis withdrawal. Forensic Toxicol 35:33-44

Klein C, Karanges E, Spiro A et al (2011) Cannabidiol potentiates Delta(9)-tetrahydrocannabinol (THC) behavioural effects and alters THC pharmacokinetics during acute and chronic treatment in adolescent rats. Psychopharmacology 218:443-457 
Kleykamp BA, Griffiths RR, Mintzer MZ (2010) Dose effects of triazolam and alcohol on cognitive performance in healthy volunteers. Exp Clin Psychopharmacol 18:1-16

Lamers CT, Ramaekers JG (2001) Visual search and urban driving under the influence of marijuana and alcohol. Hum Psychopharmacol 16: 393-401

Lenné MG, Dietze PM, Triggs TJ et al (2010) The effects of cannabis and alcohol on simulated arterial driving: influences of driving experience and task demand. Accid Anal Prev 42:859-866

Mannucci C, Navarra M, Calapai F et al (2017) Neurological aspects of medical use of Cannabidiol. CNS Neurol Disord Drug Targets 16: $541-553$

Martin-Santos R, Crippa JA, Batalla A et al (2012) Acute effects of a single, oral dose of d9-tetrahydrocannabinol (THC) and cannabidiol (CBD) administration in healthy volunteers. Curr Pharm Des 18:4966-4979

Mcleod DR, Griffiths RR, Bigelow GE et al (1982) An automated version of the Digit Symbol Substitution Test (DSST). Behav Res Methods Instrum 14:463-466

Morgan CJA, Curran HV (2008) Effects of cannabidiol on schizophrenialike symptoms in people who use cannabis. Br J Psychiatry 192: 306-307

Morgan CJ, Schafer G, Freeman TP et al (2010a) Impact of cannabidiol on the acute memory and psychotomimetic effects of smoked cannabis: naturalistic study [corrected]. Br J Psychiatry 197:285-290

Morgan CJA, Freeman TP, Schafer GL et al (2010b) Cannabidiol attenuates the appetitive effects of Delta(9)-tetrahydrocannabinol in humans smoking their chosen cannabis. Neuropsychopharmacology 35: 1879-1885

Nadulski T, Pragst F, Weinberg G et al (2005) Randomized, double-blind, placebo-controlled study about the effects of cannabidiol (CBD) on the pharmacokinetics of $\Delta 9$-tetrahydrocannabinol (THC) after oral application of THC verses standardized cannabis extract. Ther Drug Monit 27:799-810

Ogourtsova T, Kalaba M, Gelinas I et al (2018) Cannabis use and drivingrelated performance in young recreational users: a within-subject randomized clinical trial. CMAJ Open 6:E453-e462

Papafotiou K, Carter JD, Stough C (2005) The relationship between performance on the standardised field sobriety tests, driving performance and the level of Delta9-tetrahydrocannabinol (THC) in blood. Forensic Sci Int 155:172-178

Ramaekers JG (2018) Driving under the influence of cannabis: an increasing public health concern. JAMA 319:1433-1434

Ramaekers JG, Robbe H, O'Hanlon J (2000) Marijuana, alcohol and actual driving performance. Hum Psychopharmacol Clin Exp 15: $551-558$

Ramaekers JG, Kauert G, Theunissen E et al (2009) Neurocognitive performance during acute THC intoxication in heavy and occasional cannabis users. J Psychopharmacol 23:266-277

Robbe H (1998) Marijuana's impairing effects on driving are moderate when taken alone but severe when combined with alcohol. Hum Psychopharm Clin 13:S70-S78
Rogeberg O, Elvik R (2016) The effects of cannabis intoxication on motor vehicle collision revisited and revised. Addiction 111:13481359

Rogeberg O, Elvik R (2017) Response to Li et al (2017) Cannabis use and crash risk in drivers. Addiction 112:1316-1316

Roser P, Juckel G, Rentzsch J et al (2008) Effects of acute oral $\Delta 9$ tetrahydrocannabinol and standardized cannabis extract on the auditory P300 event-related potential in healthy volunteers. Eur Neuropsychopharmacol 18:569-577

Russo E, Guy GW (2006) A tale of two cannabinoids: the therapeutic rationale for combining tetrahydrocannabinol and cannabidiol. Med Hypotheses 66:234-246

Schubart CD, Sommer IEC, van Gastel WA et al (2011) Cannabis with high cannabidiol content is associated with fewer psychotic experiences. Schizophr Res 130:216-221

Schulze H, Schumacher M, Urmeew R et al (2012) DRUID final report: work performed, main results and recommendations. Bergisch Gladbach (Germany): Federal Highway Research Institute

Schwope DM, Scheidweiler KB, Huestis MA (2011) Direct quantification of cannabinoids and cannabinoid glucuronides in whole blood by liquid chromatography-tandem mass spectrometry. Anal Bioanal Chem 401:1273-1283

Spielberger CD (1983) Manual for the state-trait anxiety inventory STAI (form Y) ("self-evaluation questionnaire")

Spindle T, Cone, EJ, Schlienz, NJ et al (2018) Acute effects of smoked and vaporized cannabis in healthy adults who infrequently use cannabis: a cross-over Trial. JAMA Network Open (in press)

Stout SM, Cimino NM (2014) Exogenous cannabinoids as substrates, inhibitors, and inducers of human drug metabolizing enzymes: a systematic review. Drug Metab Rev 46:86-95

Vandrey R, Herrmann ES, Mitchell JM et al (2017) Pharmacokinetic profile of oral cannabis in humans: blood and oral fluid disposition and relation to harmacodynamic outcomes. J Anal Toxicol 41:83-99

Veldstra JL, Bosker WM, de Waard D et al (2015) Comparing treatment effects of oral THC on simulated and on-the-road driving performance: testing the validity of driving simulator drug research. Psychopharmacology 232:2911-2919

Verster JC, Roth T (2011) Standard operation procedures for conducting the on-the-road driving test, and measurement of the standard deviation of lateral position (SDLP). Int J Gen Med 4:359-371

Winton-Brown TT, Allen P, Bhattacharrya S et al (2011) Modulation of auditory and visual processing by delta-9-tetrahydrocannabinol and cannabidiol: an FMRI study. Neuropsychopharmacology 36:1340 1348

Yamaori S, Ebisawa J, Okushima Y et al (2011) Potent inhibition of human cytochrome P450 3A isoforms by cannabidiol: role of phenolic hydroxyl groups in the resorcinol moiety. Life Sci 88:730-736

Zhornitsky S, Potvin S (2012) Cannabidiol in humans - the quest for therapeutic targets. Pharmaceuticals 5:529-552

Publisher's note Springer Nature remains neutral with regard to jurisdictional claims in published maps and institutional affiliations. 